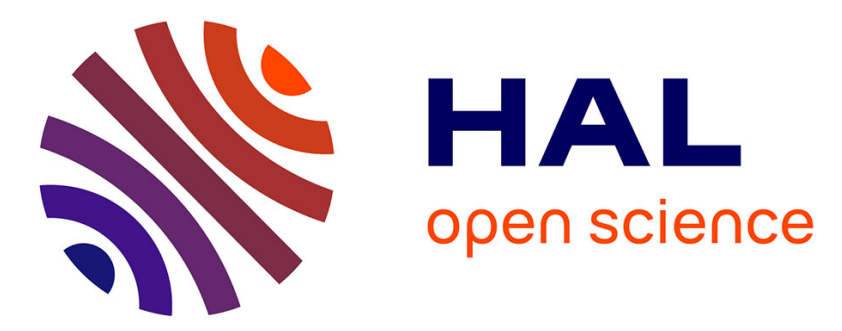

\title{
Dynamically Consistent Preferences Under Imprecise Probabilistic Information
}

Frank Riedel, Jean-Marc Tallon, Vassili Vergopoulos

\section{To cite this version:}

Frank Riedel, Jean-Marc Tallon, Vassili Vergopoulos. Dynamically Consistent Preferences Under Imprecise Probabilistic Information. 2017. halshs-01513820

\section{HAL Id: halshs-01513820 \\ https://shs.hal.science/halshs-01513820}

Preprint submitted on 25 Apr 2017

HAL is a multi-disciplinary open access archive for the deposit and dissemination of scientific research documents, whether they are published or not. The documents may come from teaching and research institutions in France or abroad, or from public or private research centers.
L'archive ouverte pluridisciplinaire HAL, est destinée au dépôt et à la diffusion de documents scientifiques de niveau recherche, publiés ou non, émanant des établissements d'enseignement et de recherche français ou étrangers, des laboratoires publics ou privés. 


\title{
PARISSCHOOL OF ECONOMICS
}

WORKING PAPER N² 2017 - 15

\author{
Dynamically Consistent Preferences \\ Under Imprecise Probabilistic Information
}

\author{
Frank Riedel \\ Jean-Marc Tallon \\ Vassili Vergopoulos
}

JEL Codes: D81

Keywords: Imprecise information, imprecision aversion, multiple priors, dynamic consistency

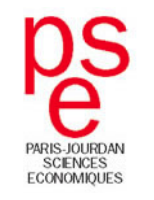




\title{
Dynamically Consistent Preferences Under Imprecise Probabilistic Information
}

\author{
Frank Riedel* $\quad$ Jean-Marc Tallon ${ }^{\dagger} \quad$ Vassili Vergopoulos ${ }^{\ddagger}$
}

April 24, 2017

\begin{abstract}
This paper extends decision theory under imprecise probabilistic information to dynamic settings. We explore the relationship between the given objective probabilistic information, an agent's subjective multiple priors, and updating. Dynamic consistency implies rectangular sets of priors at the subjective level. As the objective probabilistic information need not be consistent with rectangularity at the subjective level, agents might select priors outside the objective probabilistic information while respecting the support of the given set of priors. Under suitable additional axioms, the subjective set of priors belongs to the rectangular hull of the objective probabilistic information.
\end{abstract}

Keywords: Imprecise information, imprecision aversion, multiple priors, dynamic consistency. JEL subject classification: D81.

\section{Introduction}

Economic decisions are often made with imprecise knowledge of the statistical properties of the environment, i.e., in a situation of Knightian uncertainty. This Knightian uncertainty need not be absolute, however, as some information about possible probability distributions is usually available. In one of the famous Ellsberg (1961) experiments, for example, the

* Bielefeld University and University of Johannesburg; financial support through the German Research Foundation Grant Ri-1128-6-1 is gratefully acknowledged.

${ }^{\dagger}$ Paris School of Economics and CNRS; financial support through the Grant ANR-12-FRAL-0008-01 is gratefully acknowledged.

${ }^{\ddagger}$ Paris School of Economics and University of Paris 1 Panthéon-Sorbonne 
agent knows that the probability of drawing a red ball is one-third whereas the probability of drawing a yellow ball is anything between zero and two-thirds. Under such conditions, the agent is faced with a bet (or act) that depends on the outcome of the experiment and some imprecise information about possible probability distributions that can be described by an information set (of second order) that contains all objectively possible distributions.

Gajdos, Hayashi, Tallon, and Vergnaud (2008) (henceforth GHTV) adapted the basic analysis of Gilboa and Schmeidler (1989) (who focus solely on uncertain acts without considering information about possible probability distributions) to such uncertain environments. In this paper, we extend the axiomatic analysis of preferences under Knightian uncertainty with imprecise probabilistic information to dynamic settings.

According to GHTV, an agent who is confronted with an information set of possible priors selects a subjective set of priors and computes the worst expected utility of an act over this set of selected priors. These selected priors are consistent with the given information in the sense that their support is included in the support of objective information.

In dynamic environments, agents need to update their expectations upon the arrival of new information. Epstein and Schneider (2003) have shown that it is possible to maintain dynamic consistency for preferences over acts in a multiple-prior setting if agents update their priors in a Bayesian manner prior by prior and if the subjective set of priors is stable under pasting conditional and marginal probabilities from different priors to the original set (or, as Epstein and Schneider call it, rectangular).

In contrast to Epstein and Schneider's setting, an agent is faced with objective yet imprecise information about possible probability distributions in our setting. Ex ante, there is no reason to assume that this information is given by a rectangular set of priors. It is thus not clear how an agent should process such information or whether it is possible to maintain dynamic consistency at all.

We show here that utility functionals in the form of GHTV are dynamically consistent if the subjective set of priors is selected and updated in a suitable way. In the first place, as in GHTV's static analysis, the support of the selected set of priors has to be included in the support of the information set (i.e., the objectively known set of possible priors). In the second place, the initially chosen set has to be stable under pasting, and once the initial set of priors has been chosen, agents update their beliefs prior by prior.

An important element of our analysis is the fact that the subjective set of priors can be larger than the exogenously given set of possible priors because the agent does not want to exclude possible conditional beliefs ex ante yet also wants to be dynamically consistent. The potential "overselection" of priors is an important - albeit necessary - feature of our model.

The overselection should not be too arbitrary, though. In addition to the natural require- 
ment that the selected sets of priors be consistent with the support of objective information, we adapt two further axioms from GHTV to our dynamic setting, namely Reduction (under precise information) and Local Dominance. Local Dominance applies GHTV's dominance concept locally at each node to the next time step. When two acts are resolved in the next period and if one act is preferable to another under every element of the information set, the ranking is unchanged under the whole information set. The reduction axiom states that when the objective information consists of a single prior and this single prior is consistent with the given state of the world in that it puts mass one on the currently observed event, then the agent selects exactly this prior to evaluate acts.

The two axioms of Reduction and Local Dominance force the selected priors to be contained in the rectangular hull of the information set, i.e., the smallest rectangular set containing the initially given probabilistic information. As a first consequence, the overselection of priors does not occur in situations in which the probabilistic information is already rectangular. In other words, the overselection only emerges when the probabilistic information and the filtration are not "well-adapted" to each other. This overslection can thus be seen as an attempt on the agent's part to deal with discrepant sources of information. Second, requiring the subjective set of priors to be in the rectangular hull of the objectively given set of priors implies that Bayesian updates of the initial set of subjective priors belong to updates to the information set. In this sense, no further overselection arises at conditional stages.

Overselection is a sign of the ambiguity-averse decision maker sophistication when one is confronted with a potential conflict among sources of information that would lead to dynamically inconsistent choices. Recognizing the dynamic consistency problem, the decision maker minimally adapts his or her ex-ante preferences so as not to face consistency problems later on yet does not dismiss the ambiguous nature of the situation altogether.

There is usually a tension between dynamic consistency and deviations from expected utility. In fact, Epstein and LeBreton (1993) have shown that in order to maintain dynamic consistency along all possible information flows, it is necessary to fall back on a model of probabilistic sophistication that precludes any sensitivity to ambiguity. When the information flow is given, however, it is possible to maintain dynamic consistency for multiple priors and other ambiguity-averse models. Epstein and Schneider (2003) (see also Riedel (2004)) have shown that multiple prior preferences are dynamically consistent if each prior is updated in a Bayesian way and the set of priors is rectangular or stable under pasting marginal and conditional probabilities. Maccheroni, Marinacci, and Rustichini (2006) and Föllmer and Penner (2006) have generalized dynamic consistency to variational preferences by characterizing the suitable penalty functions for this large class of ambiguity-averse preferences.

Siniscalchi (2001) and Pires (2002) were able to consider updating for arbitrary events by weakening the notion of dynamic consistency. Siniscalchi (2011) considered a version 
of Strotz's (1955) Consistent Planning in which inconsistent agents play a dynamic game against themselves. Hanany and Klibanoff (2007) maintained both Dynamic Consistency and Relevance but allowed dynamic preferences and updated sets of priors to depend on the set of feasible plans of actions, some particular optimal plan of actions within this set, and the event that is observed. Hill (2016) observed that the incompatibility between Dynamic Consistency and Relevance only holds over objective trees and does not preclude their compatibility over subjective trees. By exploiting this idea, he developed a dynamic extension of the multiple prior model in which the ex-ante set of priors is updated on subjective contingencies in a dynamically consistent and relevant way.

The remainder of the paper is organized as follows: Section 2 briefly reviews the GHTV preferences and representation in a static decision environment. Section 3 describes the dynamic decision environment. It first presents a conditional version of Relevance and Dynamic Consistency and their characterization within dynamic GHTV preferences. It then introduces the axioms of Reduction and Local Dominance and shows how they impose restrictions on the overselection of priors. All proofs are gathered in the Appendix.

\section{The Framework}

\section{$2.1 \quad$ Objects of Choice}

Consider two nonempty sets, the outcome space $\mathcal{X}$ and the state space $S$. The state space $S$ is assumed to be finite. Let $\Delta \mathcal{X}$ denote the set of all lotteries with finite support on $\mathcal{X}$. An act is a function $f: S \rightarrow \Delta \mathcal{X}$. Let $\mathcal{F}$ stand for the set of all acts. A constant act with $f(s)=l$ for all $s \in S$ and for some lottery $l \in \Delta \mathcal{X}$ is also denoted by $l$.

Imprecise probabilistic information is modeled by a nonempty closed and convex set $P$ of probability measures on $S$. A typical element of $P$ will be denoted by $p$. Let $\mathcal{P}$ stand for the set of all nonempty, closed, and convex sets of probability measures on $S$. For $P \in \mathcal{P}$, we let $\operatorname{supp} P$ be the support of $P$ which contains all states $s \in S$ such that there exists $p \in P$ with $p(S)>0$. For a real-valued function $g: S \rightarrow \mathbb{R}$ and a probability measure $p$ on $S$, we denote by

$$
\mathbb{E}_{p}(g)=\sum_{s \in S} p(s) g(s)
$$

the expectation of $g$ under $p$.

Following GHTV, we consider uncertain acts in conjunction with imprecise probabilistic information as the basic objects of choice. Thus, an agent has a preference relation $\succsim$ defined on $\mathcal{P} \times \mathcal{F}$, the set of pairs of imprecise probabilistic information and acts. For $P, Q \in \mathcal{P}$ and $f, g \in \mathcal{F}$, the preference ranking $(P, f) \succsim(Q, g)$ means that the agent 
prefers act $f$ under probabilistic information $P$ to act $g$ under probabilistic information $Q$.

\section{$2.2 \quad$ Static representation}

We recall the static representation result of GHTV for a preference relation $\succsim$ on $\mathcal{P} \times \mathcal{F}$. Consider the following list of axioms.

Order $\succsim$ is complete and transitive. As usual, $\succ$ denotes the strict preference relation derived from $\succsim$, and $\sim$ the indifference relation.

Act Continuity For any $P \in \mathcal{P}$ and $f, g, h \in \mathcal{F}$, if $(P, f) \succ(P, g) \succ(P, h)$, there exists $\alpha, \beta \in(0,1)$ such that $(P, \alpha f+(1-\alpha) h) \succ(P, g) \succ(P, \beta f+(1-\beta) h)$.

Outcome Preference For every $P, Q \in \mathcal{P}$ and $l \in \Delta \mathcal{X},(P, l) \sim(Q, l)$.

Nontriviality There exist $P \in \mathcal{P}$ and $l, m \in \Delta \mathcal{X}$ such that $(P, l) \succ(P, m)$.

C-independence For any $P \in \mathcal{P}, f, g \in \mathcal{F}, l \in \Delta \mathcal{X}$ and $\lambda \in(0,1)$, if $(P, f) \succsim(P, g)$, then $(P, \lambda f+(1-\lambda) l) \succsim(P, \lambda g+(1-\lambda) l)$.

Uncertainty Aversion For any $P \in \mathcal{P}, f, g \in \mathcal{F}$ and $\lambda \in(0,1)$, if $(P, f) \sim(P, g)$, then $(P, \lambda f+(1-\lambda) g) \succsim(P, f)$.

Monotonicity For $P \in \mathcal{P}$ and $f, g \in \mathcal{F}$, if $(P, f(s)) \succsim(P, g(s))$ for all $s \in \operatorname{supp} P$, then $(P, f) \succsim(P, g)$.

A binary relation $\succsim$ on $\mathcal{P} \times \mathcal{F}$ is said to be imprecision averse or $G H T V$ if it satisfies the axioms Order, Act Continuity, Outcome Preference, Nontriviality, C-independence, Uncertainty Aversion and Monotonicity.

The representation theorem states that an imprecision averse decision maker uses a function $\varphi: \mathcal{P} \rightarrow \mathcal{P}$ which chooses for an objectively given set of possible distributions $P$ a set of prior $\varphi(P)$ that the agent uses to evaluate the outcomes.

Definition $1 A$ mapping $\varphi: \mathcal{P} \rightarrow \mathcal{P}$ is support-preserving if $\operatorname{supp} \varphi(P) \subseteq \operatorname{supp} P$ holds for all $P \in \mathcal{P}$. 
Theorem 1 (Gajdos, Hayashi, Tallon, and Vergnaud (2008)) A binary relation $\succsim$ on $\mathcal{P} \times \mathcal{F}$ is GHTV if and only if there exist a nonconstant linear utility function $u: \Delta \mathcal{X} \rightarrow \mathbb{R}$ and a support-preserving mapping $\varphi: \mathcal{P} \rightarrow \mathcal{P}$ such that, for any $P, Q \in \mathcal{P}$ and $f, g \in \mathcal{F}$ :

$$
(P, f) \succsim(Q, g) \Longleftrightarrow \min _{p \in \varphi(P)} \mathbb{E}_{p}(u \circ f) \geq \min _{p \in \varphi(Q)} \mathbb{E}_{p}(u \circ g)
$$

In this representation, $u$ is unique up to positive affine transformations, and $\varphi$ is unique.

Theorem 1 provides a decision-theoretic foundation to the idea that the set of priors is fully determined as a function $\varphi$ of the objective probabilistic information. In the static setting, the only restriction that $\varphi$ must satisfy is the support-preserving property; that is, only states that are deemed possible by the probabilistic information can be also deemed possible by the set of priors that the agent selects.

\section{Updating and Dynamic Representations}

\subsection{Time and Information Flow}

We consider a discrete time framework with points in time $t=0, \ldots, T$. The information flow is given by a sequence of partitions $\left(\pi_{t}\right)_{t=0, \ldots, T}$ on $S$ where $\pi_{t+1}$ refines $\pi_{t}$ for any $t=0, \ldots, T-1$. We assume that $\pi_{0}=\{S\}$. For a state $s \in S$ and a time $t=0, \ldots T$, we denote by $\pi_{t}(s)$ the unique set in $\pi_{t}$ which contains $s$. We assume that the true state of the world is revealed at time $T$. Thus, $\pi_{T}(s)=\{s\}$ for any $s \in S$.

\subsection{Basic Pointwise Representation}

We consider a family of preference relations $\left(\succsim_{t, s}\right)_{t=0, \ldots, T, s \in S}$ on $\mathcal{P} \times \mathcal{F}$. For $P, Q \in \mathcal{P}$, $f, g \in \mathcal{F}, t=0, \ldots, T$ and $s \in S$, the preference ranking $(P, f) \succsim_{t, s}(Q, g)$ means that the agent prefers act $f$ under probabilistic information $P$ to act $g$ under probabilistic information $Q$ conditional upon the event $\pi_{t}(s)$ that she observes at time $t$ and state $s$. We will use the following axioms.

GHTV For $t \in\{0, \ldots, T\}$ and $s \in S$, the binary relation $\succsim_{t, s}$ on $\mathcal{P} \times \mathcal{F}$ is GHTV.

Adaptedness For $t \in\{0, \ldots, T\}$ and $s, s^{\prime} \in S$, if $\pi_{t}(s)=\pi_{t}\left(s^{\prime}\right)$, then $\succsim_{t, s}=\succsim_{t, s^{\prime}}$. 
From the axiom GHTV and Theorem 1, we obtain a family $\left(\varphi_{t, s}\right)_{t=0, \ldots, T, s \in S}$ of supportpreserving functions $\varphi_{t, s}: \mathcal{P} \rightarrow \mathcal{P}$ and a family of nonconstant, linear (Bernoulli) utility functions $\left(u_{t, s}\right)_{t=0, \ldots, T, s \in S}$ such that the preference relation $\succsim_{t, s}$ can be represented by the utility function

$$
U_{t, s}(P, f)=\min _{p \in \varphi_{t, s}(P)} \mathbb{E}_{p}\left(u_{t, s} \circ f\right)
$$

The axiom Adaptedness requires preferences at time $t$ and state $s$ to only depend upon $s$ through the event $\pi_{t}(s)$ which the agent observes. Thus, if the agent observes the same event at two different states, the corresponding preferences at that time must be the same. A collection of functions $\left(\varphi_{t, s}\right)_{t=0, \ldots, T, s \in S}$ from $\mathcal{P}$ to $\mathcal{P}$ is said to be adapted if, for $t \in\{0, \ldots, T\}$ and $s, s^{\prime} \in S$ such that $\pi_{t}(s)=\pi_{t}\left(s^{\prime}\right)$, we have $\varphi_{t, s}=\varphi_{t, s^{\prime}}$.

Thus, for an adapted family, $\left(\varphi_{t, s}\right)_{t=0, \ldots, T, s \in S}$, the priors selected at time $t$ and state $s$ only depend upon the event which the agent observes at $(t, s)$, as well as the available probabilistic information.

We also require that the preferences over sure acts do not change over time and states.

Stable Tastes For $t, t^{\prime} \in\{0, \ldots, T\}, s, s^{\prime} \in S, P, Q \in \mathcal{P}$ and $l, m \in \Delta \mathcal{X}$, we have $(P, l) \succsim_{t, s}(Q, m)$ if and only if $(P, l) \succsim_{t^{\prime}, s^{\prime}}(Q, m)$.

As a consequence of the axiom "Stable States", the (Bernoulli) utility functions at the various times and states are positive affine transformations of each other. We can thus choose one common (Bernoulli) utility function which we simply denote by $u$. Moreover, note that the set $\varphi_{0, s}(P)$ of priors selected ex ante at any state $s \in S$ is independent of $s$ by Adaptedness and the triviality of $\pi_{0}$. We use the notation $\varphi(P)$ to refer to any of the $\varphi_{0, s}(P)$ for $s \in S$.

\subsection{Conditional Relevance}

It is natural to require that the agent's preferences do not depend on states which can be excluded at some given point in time. At time $t$ and state $s$, the agent knows that the event $\pi_{t}(s)$ happened. Given some probabilistic information, she must then be indifferent between any two acts that agree with each other on $\pi_{t}(s)$.

For any $P \in \mathcal{P}$ and $A \subseteq S$, we say that $A$ is $P$-negligible if we have $p(A)=0$ for all $p \in P$.

Conditional Relevance For $t \in\{0, \ldots, T\}$ and $s \in S, P \in \mathcal{P}$, and $f, g \in \mathcal{F}$ : if $\pi_{t}(s)$ is not $P$-negligible and $f\left(s^{\prime}\right)=g\left(s^{\prime}\right)$ for all $s^{\prime} \in \pi_{t}(s)$, then $(P, f) \sim_{t, s}(P, g)$.

We then obtain that the agent chooses only probability distributions which put full mass 
on the current information set $\pi_{t}(s)$ in the sense of the following definition.

Definition 2 A collection $\left(\varphi_{t, s}\right)_{t=0, \ldots, T, s \in S}$ of mappings from $\mathcal{P}$ to $\mathcal{P}$ is said to be conditionally relevant if, for $t \in\{0, \ldots, T\}, s \in S$, and $P \in \mathcal{P}$ such that $\pi_{t}(s)$ is not $P$-negligible, we have $p\left(\pi_{t}(s)\right)=1$ for all $p \in \varphi_{t, s}(P)$.

To understand why we restrict Conditional Relevance to situations where the observed event $\pi_{t}(s)$ is not $P$-negligible, suppose to the contrary that $\pi_{t}(s)$ is $P$-negligible. Then, supp $P \subseteq S \backslash \pi_{t}(s)$. By the support-preserving property, we must also have $\operatorname{supp} \varphi_{t, s}(P) \subseteq$ $S \backslash \pi_{t}(s)$. This implies $p\left(\pi_{t}(s)\right)=0$ for all $p \in \varphi_{t, s}(P)$ and would contradict the unrestricted versions of both Conditional Relevance and Definition 2. This shows that we need to restrict the agent's preferences only when the two sources of information are not contradictory and justifies that Conditional Relevance has bite only when these two sources are compatible.

We summarize our discussion in the following theorem.

Theorem 2 A family $\left(\succsim_{t, s}\right)_{t=0, \ldots, T, s \in S}$ of binary relations on $\mathcal{P} \times \mathcal{F}$ satisfies GHTV, Adaptedness, Conditional Relevance, and Stable Tastes if and only if there exist a nonconstant linear utility function $u: \Delta \mathcal{X} \rightarrow \mathbb{R}$ and a family $\left(\varphi_{t, s}\right)_{t=0, \ldots, T, s \in S}$ of support-preserving, adapted, and conditionally relevant mappings from $\mathcal{P}$ to itself such that $\succsim_{t, s}$ is represented by the utility function

$$
U_{t, s}(P, f)=\min _{p \in \varphi_{t, s}(P)} \mathbb{E}_{p}(u \circ f)
$$

Moreover, $u$ is unique up to positive affine transformations, and $\left(\varphi_{t, s}\right)_{t=0, \ldots, T, s \in S}$ is unique.

To have a more concise language later on, we give the following names to the list of properties of the preceding equivalence.

Definition 3 We call a family $\left(\succsim_{t, s}\right)_{t=0, \ldots, T, s \in S}$ of binary relations on $\mathcal{P} \times \mathcal{F}$ that satisfies GHTV, Adaptedness, Conditional Relevance, and Stable Tastes a Conditional Imprecision Averse Preference Family. We call a family $\left(\varphi_{t, s}\right)_{t=0, \ldots, T, s \in S}$ of support-preserving, adapted, and conditionally relevant mappings from $\mathcal{P}$ to itself a prior selection family.

Hence, the axiomatic characterization of prior selection families in terms of Conditional Imprecision Averse Preference families obtained in Theorem 2 portrays an agent selecting priors at each time $t$ and state $s$ according to $\varphi_{t, s}$. The priors selected at $(t, s)$ only deem as possible states that are already deemed possible by the probabilistic information itself. Moreover, they only depend on the probabilistic information and the event which she observes at $(t, s)$, and, when possible, assign a probability of 1 to this event. 


\subsection{Dynamic Consistency}

We now need to connect the priors selected at each state $s$ and time $t$ to one another. We thus introduce Dynamic Consistency to that effect and discuss its consequences in our model.

Dynamic Consistency For $t \in\{0, \ldots, T-1\}$ and $s \in S$, for $P \in \mathcal{P}$ such that $\pi_{t}(s)$ is not $P$-negligible, and for $f, g \in \mathcal{F}$ : If $(P, f) \succsim_{t+1, s^{\prime}}(P, g)$ for all $s^{\prime} \in \pi_{t}(s)$, then $(P, f) \succsim_{t, s}(P, g)$.

The key insight Dynamic Consistency captures is the following one: at any time $t<T$ and state $s$, if the possible future preferences at $t+1$ unanimously rank an alternative above another one, then preferences at $(t, s)$ must also rank the former one above. But note that we only require this in situations when $\pi_{t}(s)$ is not $P$-negligible; that is, when the realized event and the probabilistic information are coherent with each other.

Dynamic consistency of multiple prior representations has been studied extensively in recent years; after the basic insight of Sarin and Wakker (1998) of the role of rectangularity in a two period example, Epstein and Schneider (2003) characterize dynamic consistency for intertemporal consumption choice problems, and Delbaen (2002) and Riedel (2004) achieve the same for dynamic risk measures.

Consider a probability measure $p$ on $S$. Fix $t \in[0, T]$ and $s \in S$. If $p\left(\pi_{t}(s)\right)>0$, then, define $p_{t}(s)=p\left(\cdot \mid \pi_{t}(s)\right)$, which is another probability measure. We can also view $p_{t}(\cdot)$ as a transition kernel on $S$. Moreover, for $t<T$, define $p_{t}^{+1}(s)$ as the restriction of $p_{t}(s)$ to the algebra generated by $\pi_{t+1}$.

Consider a set $P \in \mathcal{P}$. Fix $t \in[0, T]$ and $s \in S$. If $\pi_{t}(s)$ is not $P$-negligible, then we define:

$$
P_{t}(s)=\left\{p_{t}(s), p \in P, p\left(\pi_{t}(s)\right)>0\right\} \in \mathcal{P} \quad \text { and } \quad P_{t}^{+1}(s)=\left\{p_{t}^{+1}(s), p \in P_{t}(s)\right\} .
$$

Definition 4 Fix $t \in\{0, \ldots, T-1\}$ and $s \in S$. For probability measures $p, q$ on $S$, define the pasting $p \circ_{t, s} q$ of $p$ and $q$ after $(t, s)$ as follows. If $q\left(\pi_{t+1}(s)\right)=0$, set $p \circ_{t, s} q=p$. Otherwise, we set for $s^{\prime} \in S$

$$
p \circ_{t, s} q\left(s^{\prime}\right)= \begin{cases}q\left(s^{\prime} \mid \pi_{t+1}(s)\right) p\left(\pi_{t+1}(s)\right) & \text { if } s^{\prime} \in \pi_{t+1}(s) \\ p\left(s^{\prime}\right) & \text { else. }\end{cases}
$$

For $P, Q \in \mathcal{P}$, we define their pasting after $(t, s)$ to be

$$
P \circ_{t, s} Q=\left\{p \circ_{t, s} q: p \in P, q \in Q\right\} .
$$


We call a family $\left(P_{t, s}\right)_{t=0, \ldots, T, s \in S}$ of sets of priors $P_{t, s} \in \mathcal{P}$ stable under pasting (or rectangular) if for all $t=0, \ldots, T-1$ and $s, s^{\prime} \in S$ such that $s^{\prime} \in \pi_{t}(s)$ we have

$$
P_{t, s} \circ_{t, s^{\prime}} P_{t+1, s^{\prime}}=P_{t, s} .
$$

Similarly, we call a prior selection family $\left(\varphi_{t, s}\right)_{t=0, \ldots, T, s \in S}$ stable under pasting if for all $t=0, \ldots, T-1$, all $s \in S$ such that $\pi_{t}(s)$ is not $P$-negligible and all $s^{\prime} \in \pi_{t}(s)$

$$
\varphi_{t, s}(P) \circ_{t, s^{\prime}} \varphi_{t+1, s^{\prime}}(P)=\varphi_{t, s}(P) \text {. }
$$

The pasting $p \circ_{t, s} q$ of $p$ and $q$ after $(t, s)$ describes a probability distribution whose Bayesian update on $\pi_{t+1}(s)$ agrees with that of $q$. But its Bayesian update on $S \backslash \pi_{t+1}(s)$, as well as its one-step-ahead restriction to $\left\{\pi_{t+1}(s), S \backslash \pi_{t+1}(s)\right\}$, agree with those of $p$. Such pasting can be extended to sets of probability measures. It is always possible to close a given family $\left(P_{t, s}\right)_{t=0, \ldots, T, s \in S}$ under pasting; we call the resulting family of priors the rectangular hull of $\left(P_{t, s}\right)_{t=0, \ldots, T, s \in S}$ and denote it by $\left(\operatorname{rect}_{t, s}(P)\right)_{t=0, \ldots, T, s \in S}$. More precisely, for any $s \in S$, let $\delta_{s}$ be the degenerate measure assigning a probability of 1 to state $s$. We then $\operatorname{define~} \operatorname{rect}_{t, s}(P)$ recursively for all $t=0, \ldots, T, s \in S$ and $P \in \mathcal{P}$ such that $\pi_{t}(s)$ is not $P$-negligible by setting

$$
\begin{gathered}
\operatorname{rect}_{T, s}(P):=P_{T}(s)=\left\{\delta_{s}\right\}, \text { and } \\
\operatorname{rect}_{t, s}(P):=\left\{\int_{S} p\left(s^{\prime}\right) \cdot d m\left(s^{\prime}\right), m \in P_{t}(s)^{+1}, p\left(s^{\prime}\right) \in \operatorname{rect}_{t+1, s^{\prime}}(P)\right\} .
\end{gathered}
$$

Theorem 3 A Conditional Imprecision Averse Preference Family $\left(\succsim_{t, s}\right)_{t=0, \ldots, T, s \in S}$ satisfies Dynamic Consistency if and only if the prior selection family $\left(\varphi_{t, s}\right)_{t=0, \ldots, T, s \in S}$ in the representation (2) is stable under pasting. Moreover, when these equivalent conditions hold, we further have:

(1) For any $t \in\{0, \ldots, T-1\}, s \in S, P \in \mathcal{P}$ such that $\pi_{t}(s)$ is not P-negligible, and any $f \in \mathcal{F}$,

$$
U_{t, s}(P, f)=\min _{m \in \varphi_{t, s}(P)^{+1}}\left\{\int_{S} U_{t+1, s^{\prime}}(P, f) \cdot d m\left(s^{\prime}\right)\right\}
$$

(2) For any $t \in\{0, \ldots, T-1\}, s \in S$ and $P \in \mathcal{P}$ such that $\pi_{t}(s)$ neither $P$-negligible nor $\varphi(P)$-negligible,

$$
\varphi_{t, s}(P)=\varphi(P) \mid \pi_{t}(s) \equiv\left\{p\left(. \mid \pi_{t}(s)\right) \mid p\left(\pi_{t}(s)\right)>0 \text { and } p \in \mathcal{P}\right\},
$$

Theorem 3 characterizes Dynamic Consistency for Conditional Imprecision Averse Preference families in terms of the stability under pasting of the prior selection family. Moreover, 
Equation (3) shows that Dynamic Consistency leads to value functions $\left\{U_{t, s}, t=0, \ldots, T\right.$, $s \in S\}$ with a recursive structure. This property lies at the heart of dynamic programming methods, as it ensures the equality between backward induction solutions and ex-ante optimal plans. It also secures that information has always a nonnegative value.

In addition, Theorem 3 also shows through Equation (4) that the Full Bayes updating rule is implied: given some objective information $P$, the priors that are selected at $(t, s)$ consist of the Bayesian updates on the available event $\pi_{t}(s)$ of all the priors selected ex ante under $P$. Thus, it is still true, as in Theorem 2 , that the priors selected at $(t, s)$ only depend on the probabilistic information $P$ and the available event $\pi_{t}(s)$. Equation (4) clarifies that these priors only depend on $P$ through the set $\varphi(P)$ of priors selected ex ante. In this sense, the priors selected at $(t, s)$ can also be seen as fully determined by the ex-ante priors (under the same objective information) and the available event.

Theorem 3 leaves a lot of freedom for the choice of $\varphi(P)$. In light of GHTV's Theorem 2, one might expect the ex-ante prior selection process to satisfy the following property: $\varphi(P) \subseteq P$ for any $P \in \mathcal{P}$. GHTV call this the selection property. It would mean that the agent selects her ex-ante priors within the available objective information. But the next example shows that this is too restrictive as it sometimes implies neutrality to ambiguity, an undesirable feature.

Example 1 Consider the following dynamic version of the Ellsberg (1961) experiment. An agent takes bets on the color of the ball drawn from an urn containing 90 balls of three possible colors: red, blue and green. The state space is $S=\{r, b, g\}$. Objective information takes the form of a set of probability measures $P_{a, b}=\{(1 / 3, p, 2 / 3-p), a \leq p \leq b\}$ for some $a, b$ with $0 \leq a \leq b \leq 2 / 3$. Each of these sets conveys the information that exactly 30 of the balls are red, together with a more or less imprecise estimation of the numbers of blue and green balls. Moreover, the agent will be told whether the drawn ball is green or not. Thus, the information flow is determined by the partition $\pi=\{E, F\}$ where $E=\{r, b\}$ and $F=\{g\}$.

Then, fix $a_{E}, b_{E}, \underline{m}, \bar{m} \in[0,1]$ possibly depending on a and $b$ with $a_{E} \leq b_{E}$ and $\underline{m} \leq \bar{m}$. Define

$$
\begin{gathered}
\varphi_{E}\left(P_{a, b}\right)=\left\{(p, 1-p, 0), a_{E} \leq p \leq b_{E}\right\} \quad \text { and } \quad \varphi_{F}\left(P_{a, b}\right)=\{(0,0,1)\}, \\
\varphi_{0}\left(P_{a, b}\right)=\left\{\left(\left(\frac{1}{3}+m\right) p,\left(\frac{1}{3}+m\right)(1-p), \frac{2}{3}-m\right), a_{E} \leq p \leq b_{E}, \underline{m} \leq m \leq \bar{m}\right\} .
\end{gathered}
$$

The collection $\left.\left.\left\{\varphi_{0}\left(P_{a, b}\right), \varphi_{E}\left(P_{a, b}\right)\right), \varphi_{F}\left(P_{a, b}\right)\right)\right\}$ is stable under pasting for any $a, b$, consistently with Theorem $3^{1}$

\footnotetext{
${ }^{1}$ We abuse slightly notation here. Note that time is here always $t+0=0$ or $t=1$. By Stable Tastes, $\varphi_{0, s}$ is independent of $s$, and denoted $\varphi(P)$. By Adaptedness, we can identify $\varphi_{1, s}$ is constant over $E$ (resp. $F)$, and denoted $\varphi_{E}\left(\right.$ resp. $\left.\varphi_{F}\right)$.
} 
It might be tempting to choose $a_{E}, b_{E}, \underline{m}, \bar{m} \in[0,1]$ so as to have $\varphi_{0}\left(P_{a, b}\right) \subseteq P_{a, b}$ for any $a, b$. However, for any mappings $\varphi_{0}, \varphi_{E}$ and $\varphi_{F}$ such that $\left.\left.\left\{\varphi_{0}\left(P_{a, b}\right), \varphi_{E}\left(P_{a, b}\right)\right), \varphi_{F}\left(P_{a, b}\right)\right)\right\}$ is stable under pasting and $\varphi_{0}\left(P_{a, b}\right) \subseteq P_{a, b}$, we have that $\varphi_{0}\left(P_{a, b}\right)$ is a singleton. Indeed, suppose that $(1 / 3, p, 2 / 3-p)$ and $(1 / 3, q, 2 / 3-q)$ belong to $\varphi_{0}\left(P_{a, b}\right)$. Then, by stability under pasting,

$$
\left(\frac{\frac{1}{3}+p}{1+3 q}, \frac{3 q\left(\frac{1}{3}+p\right)}{1+3 q}, 2 / 3-p\right)=(1 / 3, p, 2 / 3-p) \circ_{E}(1 / 3, q, 2 / 3-q) \in \varphi_{0}\left(P_{a, b}\right) \subseteq P_{a, b} .
$$

But then $(1 / 3+p) /(1+3 q)=1 / 3$ and, therefore, $p=q$. Hence, imposing that the selected priors be a subset of the set $P$ has the overly strong implication in this example to impose that the agent is neutral to the ambiguity of the situation captured by the fact that $P$ is not a singleton.

Ambiguity is typically attributed to the "poor" quality of the probabilistic information that an agent has. Thus, one could interpret the fact that the selection property implies ambiguity neutrality in cases where the objective information $P$ does not contain nontrivial rectangular subsets as meaning that these sets $P$ represent information of "good" quality, or are "falsely ambiguous". This interpretation however is not satisfactory, as such sets $P$ can still be very dispersed. In the example, $P_{0, \frac{2}{3}}$ contains no nontrivial rectangular subset but is still imprecise enough and generates ambiguity as demonstrated by the Ellsberg (1961) paradox itself.

Our view is therefore that overselection of priors, when the original set does not contain nontrivial rectangular subsets, is a desirable feature, as it does not force the decision criterion to be expected utility in these circumstances. We further investigate the way the selection operates in the next section.

Last, a further understanding of the way selection operates in our context is to explore the consequence of requiring that $\varphi(P)=\varphi(\operatorname{rect}(P))$ for any $P \in \mathcal{P}$. This would mean that the agent always "rectangularizes" the objective probabilistic information that he is given to make it fit well with the structure of the information flow of events.

But consider now the particular case where the filtration consists of a single partition $\pi$. Suppose that $P$ is conditionally more precise than $Q$ in the sense of GHTV (with respect to $\pi)$. Then, $P$ and $Q$ have the same rectangular hull. So the condition implies $\varphi(P)=\varphi(Q)$. In turn, given the representation obtained in Theorem 3 , this implies that $(P, f)$ and $(Q, f)$ must be indifferent for any $f$. In the terminology of GHTV, an agent whose prior selection family satisfies the condition is thus necessarily indifferent to imprecision.

Hence, an agent who is strictly averse to imprecision cannot satisfy the condition. This means that the selected priors do not only depend upon the rectangular hull of $P$ under the filtration: the selected priors depend also on the "specific nonrectangular shape" of 
$P$, that is, on the way $P$ provides hedging for the ambiguity perceived conditionally at disjoint events.

\subsection{Local Dominance}

A consequence of Theorem 3 is that an agent with preferences satisfying Dynamic Consistency and Conditional Relevance and revealing a nonneutral attitude towards ambiguity must sometimes select her ex-ante priors outside the probabilistic information that she disposes of. So far, the only restriction on this overselection is the support-preserving property of the prior selection family: the priors selected ex ante must only assign positive probability weight to states already receiving a positive weight from the probabilistic

information. Any prior is thus not allowed. We now develop other restrictions for this overselection.

The Dominance criterion employed by GHTV restricts the agent's choice of priors to subsets of $P$. We will now impose a weaker and local version of this dominance criterion that is suited to our dynamic framework.

Local Dominance For $t \in\{0, \ldots, T-1\}, s \in S, P \in \mathcal{P}$ such that $\pi_{t}(s)$ is not $P$ negligible, and $f, g \in \mathcal{F}$ that are $\pi_{t+1}$-measurable: if $(\{p\}, f) \succsim t, s(\{p\}, g)$ for all $p \in P_{t}(s)$, then $(P, f) \succsim_{t, s}(P, g)$.

Because of the role played by the Bayesian updating of the objective information on the events in the filtration, Local Dominance can be understood as a requirement of consistency between preferences and the two sources of information, the objective probabilistic set and the filtration. Moreover, Local Dominance can be also seen as a criterion of internal consistency of the preference relation $\succsim_{t, s}$ at some pair $(t, s)$ : if act $f$ is at least as good as act $g$ under the Bayesian update on $\pi_{t}(s)$ of any of the probability distributions in $P$, then $f$ must also be at least as good as $g$ under $P$. But we only require this for acts $f$ and $g$ are measurable with respect to the partition of the next stage. Omitting this restriction would lead to the inclusion $\varphi(P) \subseteq P$ for any $P \in \mathcal{P}$ (See GHTV's Theorem 2), which as explained would be too strong for our purposes. In fact, the dominance reasoning captured in the axiom becomes questionable when applied to nonmeasurable acts: since the uncertainty attached to these acts is not fully resolved at the next stage, the ambiguities perceived at the disjoint cells of the next stage partition might hedge one another and explain failures of the dominance reasoning. The measurability restriction is thus meant to allow such hedging to play a role in decisions.

We will also use a version of the criterion of Reduction under Precise Information employed by GHTV. But it requires additional notation. Fix $p \in \Delta S$ and $f \in \mathcal{F}$. Then, there is a partition $\left(E_{1}, \ldots, E_{n}\right)$ and a collection $\left(l_{1}, \ldots, l_{n}\right)$ of lotteries on $\mathcal{X}$ such that $f(s)=l_{i}$ for any 
$s \in E_{i}$ and any $i \in[1, n]$. Then, define $l(p, f) \in \Delta \mathcal{X}$ as the lottery given by $\sum_{i=1}^{n} p\left(E_{i}\right) \cdot l_{i}$. Note that this definition is independent of the specific partition $\left(E_{1}, \ldots, E_{n}\right)$ that is chosen to construct $l(p, f)$.

Reduction For any $t \in\{0 \ldots T-1\}$, any $s \in S$, and for any $p \in \Delta S$ such that $p\left(\pi_{t}(s)\right)=1$, we have $(\{p\}, f) \sim_{t, s}(\{p\}, l(p, f))$, for any $f \in \mathcal{F}$.

Under the Reduction axiom, whenever the objective information consists of a single probability measure that is consistent with the available event, the selected priors must be that measure as captured by the following definition.

Definition 5 A prior selection family $\left(\varphi_{t, s}\right)_{t=0, \ldots, T, s \in S}$ is said to be grounded if $\varphi_{t, s}(\{p\})=$ $\{p\}$ for any $p \in \Delta S$ such that $p\left(\pi_{t}(s)\right)=1$, and any $t \in[0, T]$ and $s \in S$.

The next theorem uses Local Dominance and Reduction to further constrain the selection of priors.

Theorem 4 A Conditional Imprecision Averse Preference Family $\left(\succsim_{t, s}\right)_{t=0, \ldots, T, s \in S}$ satisfies Dynamic Consistency, Local Dominance and Reduction if and only if the prior selection family $\left(\varphi_{t, s}\right)_{t=0, \ldots, T, s \in S}$ in the representation (2) is stable under pasting, grounded and we have

$$
\varphi_{t, s}(P) \subseteq \operatorname{rect}_{t, s}(P),
$$

for all $t \in\{0, \ldots, T\}, s \in S$, and $P \in \mathcal{P}$ such that $\pi_{t}(s)$ is not $P$-negligible.

In the dynamic setting, the probabilistic information $P$ might be given in a way that does not fit well with the structure of the information flow of states of nature in the sense that $P$ itself is not stable under pasting (or rectangular) with respect to the filtration of events. Equation (5) shows that the agent "rectangularizes" the probabilistic information (or closes it under pasting according to the given information flow). In other words, she chooses freely her priors within the rectangular hull of $P$. Note that this is always consistent with the support-preserving property of the prior selection family as the rectangularization itself preserves the support of a set of measures.

Confining the additional priors within the rectangular hull of probabilistic information has at least two advantages. First, if the objective information $P$ fits well the structure of the information flow, then $P$ is already rectangular. In particular, we obtain $\varphi(P) \subseteq P$. Thus, the agent is only allowed to select ex-ante priors outside the objective information in situations where the latter is not well-adapted to the filtration. Second, the rectangular 
hull of a set $P$ does never add new posteriors conditional on the events in the filtration. Therefore, at any $(t, s)$ such that $\pi_{t}(s)$ is a proper and not $P$-negligible subset of $S$, we have $\varphi_{t, s}(P) \subseteq P_{t}(s)$. Thus, all the selected priors must be Bayesian updates on the available event of measures in the objective information. Hence, overselection is here the natural consequence of the decision maker's desire to act in a dynamically consistent manner while acknowledging the imprecision of the information he has.

Getting back to the dynamic version of the Ellsberg (1961) experiment, the rectangular hull of the sets $P_{a, b}$ is given by:

$$
\begin{gathered}
\operatorname{rect}_{E}\left(P_{a, b}\right)=\left\{\left(\frac{1}{(1+3 p)}, \frac{3 p}{(1+3 p)}, 0\right), a \leq p \leq b\right\} \quad \text { and } \operatorname{rect}_{F}\left(P_{a, b}\right)=\{(0,0,1)\}, \\
\operatorname{rect}_{0}\left(P_{a, b}\right)=\left\{\left(\frac{\frac{1}{3}+m}{(1+3 p)}, \frac{\left(\frac{1}{3}+m\right) 3 p}{(1+3 p)}, \frac{2}{3}-m\right), a \leq m, p \leq b\right\}
\end{gathered}
$$

Now, for any $a, b$, let $a^{\prime}, b^{\prime} \in[0,1]$, possibly depending on $a$ and $b$, be such that $a \leq a^{\prime} \leq$ $b^{\prime} \leq b$. Then, define $\left.\left.\left(\varphi_{0}\left(P_{a, b}\right), \varphi_{E}\left(P_{a, b}\right)\right), \varphi_{F}\left(P_{a, b}\right)\right)\right)$ according to:

$$
\begin{aligned}
\varphi_{E}\left(P_{a, b}\right)= & \left\{\left(\frac{1}{(1+3 p)}, \frac{3 p}{(1+3 p)}, 0\right), a^{\prime} \leq p \leq b^{\prime}\right\} \quad \text { and } \quad \varphi_{F}\left(P_{a, b}\right)=\{(0,0,1)\}, \\
& \varphi_{0}\left(P_{a, b}\right)=\left\{\left(\frac{\left(\frac{1}{3}+m\right)}{(1+3 p)}, \frac{\left(\frac{1}{3}+m\right) 3 p}{(1+3 p)}, \frac{2}{3}-m\right), a^{\prime} \leq m, p \leq b^{\prime}\right\}
\end{aligned}
$$

By construction, $\left.\left.\left\{\varphi_{0}\left(P_{a, b}\right), \varphi_{E}\left(P_{a, b}\right)\right), \varphi_{F}\left(P_{a, b}\right)\right)\right\}$ is stable under pasting and satisfies Equation (5) for any $a, b$, consistently with Theorem 4.

\section{Appendix}

\section{Proof of Theorem 3}

By Theorem 2, there exist a nonconstant linear utility function $u: \Delta \mathcal{X} \rightarrow \mathbb{R}$ and a family $\left(\varphi_{t, s}\right)_{t=0, \ldots, T, s \in S}$ of support-preserving, adapted, and conditionally relevant mappings from $\mathcal{P}$ to itself such that for any $t \in\{0, \ldots, T\}$ and $s \in S$, and for any $P, Q \in \mathcal{P}$ and $f, g \in \mathcal{F}$ :

$$
(P, f) \succsim_{t, s}(Q, g) \Longleftrightarrow U_{t, s}(P, f) \geq U_{t, s}(Q, g),
$$

where, for any $P \in \mathcal{P}$ and $f \in \mathcal{F}$, we have:

$$
U_{t, s}(P, f)=\min _{p \in \varphi_{t, s}(P)} \mathbb{E}_{p}(u \circ f)
$$


Now, fix $t \in\{0, \ldots, T-1\}, s \in S$ and $P \in \mathcal{P}$ such that $\pi_{t}(s)$ is not $P$-negligible. Moreover, fix $f \in \mathcal{F}$. Note that it is simple to obtain the following fact: For $t^{\prime} \in[0, T]$ and $s^{\prime} \in S$, there exists $l_{t^{\prime}, s^{\prime}} \in \Delta \mathcal{X}$ such that $(P, f) \sim_{t^{\prime}, s^{\prime}}\left(P, l_{t^{\prime}, s^{\prime}}\right)$. We can further assume without loss of generality that we have $l_{t^{\prime}, s^{\prime}}=l_{t^{\prime \prime}, s^{\prime \prime}}$ whenever $\succsim_{t^{\prime}, s^{\prime}}=\succsim_{t^{\prime \prime}, s^{\prime \prime}}$. Let us consider the act $g \in \mathcal{F}$ defined by $g\left(s^{\prime}\right)=l_{t+1, s^{\prime}}$ for any $s^{\prime} \in S$. Since $\pi_{t}(s)$ is not $P$-negligible, it is also true that $\pi_{t}\left(s^{\prime}\right)$ is not $P$-negligible for any $s^{\prime} \in \pi_{t}(s)$. We can apply Conditional Relevance and obtain $(P, g) \sim_{t+1, s^{\prime}}\left(P, l_{t+1, s^{\prime}}\right)$ for any $s^{\prime} \in \pi_{t}(s)$. This gives $(P, g) \sim_{t+1, s^{\prime}}(P, f)$ for any $s^{\prime} \in \pi_{t}(s)$. Then, by Dynamic Consistency, $(P, g) \sim_{t, s}(P, f)$. Therefore, by Equation (6), and using the $\pi_{t+1}$-measurability of $g$,

$$
\min _{p \in \varphi t, s}(P) \mathbb{E}_{p}(u \circ f)=\min _{p \in \varphi t, s}(P) \mathbb{E}_{p}(u \circ g)=\min _{m \in \varphi_{t, s}(P)^{+1}}\left\{\int_{S} u\left(l_{t+1, s^{\prime}}\right) \cdot d m\left(s^{\prime}\right)\right\}
$$

Meanwhile, given the definition of $l_{t+1, s^{\prime}}$ as well as the representation obtained in Equation (6), we have

$$
u\left(l_{t+1, s^{\prime}}\right)=\min _{p \in \varphi_{t+1, s^{\prime}}(P)} \mathbb{E}_{p}(u \circ f)
$$

Therefore, combining Equation (8) and (9), we obtain:

$$
\min _{p \in \varphi_{t, s}(P)} \mathbb{E}_{p}(u \circ f)=\min _{m \in \varphi_{t, s}(P)^{+1}}\left\{\int_{S} \min _{p \in \varphi_{t+1, s^{\prime}}(P)} \mathbb{E}_{p}(u \circ f) \cdot d m\left(s^{\prime}\right)\right\}
$$

Hence, we have the dynamic programming principle of Equation (3). As a consequence, we can now also write

$$
\min _{p \in \varphi_{t, s}(P)} \mathbb{E}_{p}(u \circ f)=\min _{p \in \tilde{\varphi}_{t, s}(P)} \mathbb{E}_{p}(u \circ f),
$$

where $\tilde{\varphi}_{t, s}(P)$ is the closed and convex set defined by

$$
\tilde{\varphi}_{t, s}(P)=\left\{\int_{S} p\left(s^{\prime}\right) \cdot d m\left(s^{\prime}\right), m \in \varphi_{t, s}(P)^{+1}, p\left(s^{\prime}\right) \in \varphi_{t+1, s^{\prime}}(P)\right\} .
$$

By the uniqueness part of the Gilboa and Schmeidler (1989) theorem, we obtain $\varphi_{t, s}(P)=$ $\tilde{\varphi}_{t, s}(P)$. As a result, for any $t \in\{0, \ldots, T-1\}, s \in S$ and $P \in \mathcal{P}$ such that $\pi_{t}(s)$ is not $P$-negligible, we have

$$
\varphi_{t, s}(P)=\left\{\int_{S} p\left(s^{\prime}\right) \cdot d m\left(s^{\prime}\right), m \in \varphi_{t, s}(P)^{+1}, p\left(s^{\prime}\right) \in \varphi_{t+1, s^{\prime}}(P)\right\} .
$$

Furthermore, for any state $s^{\prime} \in \pi_{t}(s)$ such that $\pi_{t+1}\left(s^{\prime}\right)$ is not $\varphi_{t, s}(P)$-negligible, Equation (13) gives

$$
\varphi_{t+1, s^{\prime}}(P)=\varphi_{t, s}(P) \mid \pi_{t+1}\left(s^{\prime}\right)
$$


We now show that the prior selection family $\left(\varphi_{t, s}\right)_{t=0, \ldots, T, s \in S}$ is stable under pasting. Let $t \in\{0, \ldots, T-1\}, s \in S$ and $P \in \mathcal{P}$ such that $\pi_{t}(s)$ is not $P$-negligible. It is sufficient to show $\varphi_{t, s}(P) \circ_{t, s^{\prime}} \varphi_{t+1, s^{\prime}}(P)=\varphi_{t, s}(P)$ for any $s^{\prime} \in \pi_{t}(s)$.

First, take $p \in \varphi_{t, s}(P)$. If $p\left(\pi_{t+1}\left(s^{\prime}\right)\right)>0$, then $\pi_{t+1}\left(s^{\prime}\right)$ is not $\varphi_{t, s}(P)$-negligible and, by Equation (14), we have $p\left(. \mid \pi_{t+1}\left(s^{\prime}\right)\right) \in \varphi_{t+1, s^{\prime}}(P)$ and $p=p \circ_{t, s^{\prime}} p\left(. \mid \pi_{t+1}\left(s^{\prime}\right)\right) \in \varphi_{t, s}(P) \circ_{t, s^{\prime}}$ $\varphi_{t+1, s^{\prime}}(P)$. If $p\left(\pi_{t+1}\left(s^{\prime}\right)\right)=0$. Then, $p=p \circ_{t, s^{\prime}} q$ for any measure $q$ on $S$. So it is sufficient to take $q \in \varphi_{t+1, s^{\prime}}(P)$ to obtain $p \in \varphi_{t, s}(P) \circ_{t, s^{\prime}} \varphi_{t+1, s^{\prime}}(P)$.

Now, take $p \in \varphi_{t, s}(P) \circ_{t, s^{\prime}} \varphi_{t+1, s^{\prime}}(P)$. So $p=m \circ_{t, s^{\prime}} q$ with $m \in \varphi_{t, s}(P)$ and $q \in \varphi_{t+1, s^{\prime}}(P)$. Then, $p$ must be an element of $\tilde{\varphi}_{t, s}(P)$ and, therefore, of $\varphi_{t, s}(P)$.

Moreover, an induction on $t \in[0, T]$ relying upon Equation (14) finally shows the following equality: for any $t \in[0, T], s \in S$ and $P \in \mathcal{P}$ such that $\pi_{t}(s)$ neither $P$-negligible nor $\varphi(P)$-negligible,

$$
\varphi_{t, s}(P)=\varphi(P) \mid \pi_{t}(s)
$$

Now assume that the prior selection family $\left(\varphi_{t, s}\right)_{t=0, \ldots, T, s \in S}$ in the representation (2) is stable under pasting. Fix $t \in\{0, \ldots, T-1\}, s \in S$ and $P \in \mathcal{P}$ such that $\pi_{t}(s)$ is not $P$-negligible. By iterative applications of stability under pasting, we obtain the equality $\varphi_{t, s}(P)=\tilde{\varphi}_{t, s}(P)$. This entails in turn that Equation (10) holds for any $f \in \mathcal{F}$. From there, dynamic consistency easily follows.

\section{Proof of Theorem 4}

First assume Dynamic Consistency, Local Dominance and Reduction. Let $u,\left(\varphi_{t, s}\right)_{t=0, \ldots, T, s \in S}$ and $\left(U_{t, s}\right)_{t=0, \ldots, T, s \in S}$ be as in Theorem 3. Let us now use Reduction to show groundedness; that is, we show that $\varphi_{t, s}(\{p\})=\{p\}$ for any $p \in \Delta S$ such that $p\left(\pi_{t}(s)\right)=1$, and any $t \in[0, T]$ and $s \in S$. By Reduction and linearity, for any $f \in \mathcal{F}, U_{t, s}(\{p\}, f)=$ $U_{t, s}(\{p\}, l(p, f))=u(l(p, f))=\sum_{i=1}^{n} u\left(l_{i}\right) p\left(E_{i}\right)=\mathbb{E}_{p}(u \circ f)$. Therefore, by the uniqueness part of Gilboa and Schmeidler's theorem (1989), we obtain $\varphi_{t, s}(\{p\})=\{p\}$.

Now, fix $P \in \mathcal{P}$ and $s \in S$ such that $P_{T}(s) \neq \varnothing$. We show that $\varphi_{T, s}(P) \subseteq P_{T}(s)$. Since $P_{T}(s) \neq \varnothing$, we have that $\pi_{T}(s)=\{s\}$ is not $P$-negligible. Since the prior selection family is conditionally relevant, any prior in $\varphi_{T, s}(P)$ puts a probability of 1 on $\{s\}$. Therefore, $\varphi_{T, s}(P)$ only contains the Dirac distribution at $s$. Similarly for $P_{T}(s)$. Hence the inclusion $\varphi_{T, s}(P) \subseteq P_{T}(s)$.

Moreover, we show that, for any $P \in \mathcal{P}, s \in S$ and $t<T$ such that $P_{t}(s) \neq \varnothing$, we have $\varphi_{t, s}(P)^{+1} \subseteq P_{t}^{+1}(s)$ by means of contradiction. If $\varphi_{t, s}(P)^{+1} \nsubseteq P_{t}^{+1}(s)$, then there exists $p^{*} \in \varphi_{t, s}(P)^{+1}$ such that $p^{*} \notin P_{t}^{+1}(s)$. By the separation theorem, we obtain an 
$\pi_{t+1}$-measurable function $F: S \rightarrow \mathbb{R}$ such that:

$$
\min _{p \in \varphi, s}(P)^{+1} \mathbb{E}_{p}(F) \leq \mathbb{E}_{p^{*}}(F)<\min _{p \in P_{t}^{+1}(s)} \mathbb{E}_{p}(F)
$$

Without loss of generality, we assume that $F$ is of norm less than 1. By adequately normalizing $u$ if necessary, we can also assume that the range of $u$ contains $[-1,1]$. So $F$ is necessarily of the form $F=u \circ f$, for some $\pi_{t+1}$-measurable $f \in \mathcal{F}$. Therefore, Equation (16) becomes:

$$
U_{t, s}(P, f) \leq \mathbb{E}_{p^{*}}(u \circ f)<\min _{p \in P_{t}^{+1}(s)} \mathbb{E}_{p}(u \circ f)
$$

Now, define another $\pi_{t+1}$-measurable $g=(1 / 2) f+(1 / 2) l \in \mathcal{F}$ where $l \in \Delta \mathcal{X}$ is defined by $l=l\left(p^{*}, f\right)$ and, therefore, satisfies:

$$
u(l)=\mathbb{E}_{p^{*}}(u \circ f)
$$

On the one hand, for any $p \in P_{t}(s)$, we have $\varphi_{t, s}(\{p\})=\{p\}$. So $U_{t, s}(\{p\}, g)=\mathbb{E}_{p}(u \circ g)=$ $(1 / 2) \mathbb{E}_{p}(u \circ f)+(1 / 2) u(l)=(1 / 2) \mathbb{E}_{p}(u \circ f)+(1 / 2) \mathbb{E}_{p^{*}}(u \circ f) \leq \mathbb{E}_{p}(u \circ f)=U_{t, s}(\{p\}, f)$ by Equation (17). Thus, $(\{p\}, f) \succsim_{t, s}(\{p\}, g)$ for any $p \in P_{t}(s)$.

On the other hand, $U_{t, s}(P, g)=(1 / 2) U_{t, s}(P, f)+(1 / 2) u(l)=(1 / 2) U_{t, s}(P, f)+(1 / 2) \mathbb{E}_{p^{*}}(u \circ$ $f) \geq U_{t, s}(P, f)$ by Equation (17). Thus, $(P, g) \succsim_{t, s}(P, f)$. But then Local Dominance is contradicted, which finally shows that $\varphi_{t, s}(P)^{+1} \subseteq P_{t}^{+1}(s)$.

Moreover, since Dynamic Consistency holds, we can proceed as in the proof of Theorem 3 to obtain the equality $\varphi_{t, s}(P)=\tilde{\varphi}_{t, s}(P)$ for any $t \in\{0, \ldots, T-1\}, s \in S$ and $P \in \mathcal{P}$ such that $\pi_{t}(s)$ is not $P$-negligible.

Now we use these facts to show by induction that for any $t \in[0, T]$ and $s \in S$ such that $P_{t}(s) \neq \varnothing$

$$
\varphi_{t, s}(P) \subseteq \operatorname{rect}_{t, s}(P)
$$

First, if $t=T$, we have $\varphi_{T, s}(P) \subseteq P_{T}(s)$ which shows (19). If $t=T-1$, we have

$$
\varphi_{T-1, s}(P)=\tilde{\varphi}_{T-1, s}(P)=\left\{\int_{S} p\left(s^{\prime}\right) \cdot d m\left(s^{\prime}\right), m \in \varphi_{T-1, s}(P)^{+1}, p\left(s^{\prime}\right) \in \varphi_{T, s^{\prime}}(P)\right\}
$$

But we know that $\varphi_{T-1, s}(P)^{+1} \subseteq P_{T-1}^{+1}(s)$ if $P_{T-1}(s) \neq \varnothing$ and $\varphi_{T, s^{\prime}}(P) \subseteq P_{T}\left(s^{\prime}\right)$. Therefore, we obtain

$$
\varphi_{T-1, s}(P) \subseteq\left\{\int_{S} p\left(s^{\prime}\right) \cdot d m\left(s^{\prime}\right), m \in P_{T-1}(s)^{+1}, p\left(s^{\prime}\right) \in P_{T}\left(s^{\prime}\right)\right\}=\operatorname{rect}_{T-1, s}(P)
$$

To complete the proof in the case where $t<T$, we proceed as in the case where $t=T-1$. As for the necessity of the axioms, Reduction follows directly from the fact that $\left(\varphi_{t, s}\right)_{t \in[0, T]}^{s \in S}$ 
is grounded. Last, to show Local Dominance, first note that, since $P_{t}(s) \neq \varnothing$, we have $\varphi_{t, s}(P) \subseteq \operatorname{rect}_{t, s}(P)$. Thus, $\varphi_{t, s}(P)^{+1} \subseteq \operatorname{rect}_{t, s}(P)^{+1}=P_{t}(s)^{+1}$. Now, take $f, g \in \mathcal{F}$ that are $\pi_{t+1}$-measurable such that $(\{p\}, f) \succsim_{t, s}(\{p\}, g)$ for any $p \in P_{t}(s)$. Since $\varphi_{t, s}(\{p\})=\{p\}$ (by $p \in P_{t}(s)$ and groundedness), $\mathbb{E}_{p}(u \circ f) \geq \mathbb{E}_{p}(u \circ g)$ for any $p \in P_{t}(s)$ and, therefore, for any $p \in \varphi_{t, s}(P)^{+1} \subseteq P_{t}(s)^{+1}$. But then, since $f, g$ are $\pi_{t+1}$-measurable, we have $\mathbb{E}_{p}(u \circ f) \geq \mathbb{E}_{p}(u \circ g)$ for any $p \in \varphi_{t, s}(P)$. This finally shows $U_{t, s}(P, f) \geq U_{t, s}(P, f)$. Hence Local Dominance.

\section{References}

Delbaen, F. (2002): "Coherent risk measures on general probability spaces'," Advances in finance and stochastics, pp. 1-37.

EllsberG, D. (1961): "Risk, ambiguity, and the Savage axioms," Quarterly Journal of Economics, 75, 643-669.

Epstein, L., And M. LeBreton (1993): "Dynamically consistent beliefs must be Bayesian," Journal of Economic Theory, 61(1), 1-22.

Epstein, L., And M. Schneider (2003): "Recursive multiple prior," Journal of Economic Theory, 113, 1-31.

Föllmer, H., ANd I. Penner (2006): "Convex risk measures and the dynamics of their penalty functions," Statistics and Decisions, 24(1), 61-96.

Gajdos, T., T. Hayashi, J.-M. Tallon, and J.-C. Vergnaud (2008): "Attitude toward imprecise information," Journal of Economic Theory, 140, 23-56.

Gilboa, I., And D. Schmeidler (1989): "Maxmin expected utility with a non-unique prior," Journal of Mathematical Economics, 18, 141-153.

Hanany, E., And P. Klibanoff (2007): "Updating preferences with multiple priors," Theoretical Economics, 2(3), 261-298.

Hill, B. (2016): "Dynamic Consistency and Ambiguity: A Reappraisal," mimeo.

Maccheroni, F., M. Marinacci, and A. Rustichini (2006): "Dynamic variational preferences," Journal of Economic Theory, 128(1), 4-44.

Pires, C. (2002): "A Rule For Updating Ambiguous Beliefs," Theory and Decision, 53(2), $137-152$.

Riedel, F. (2004): "Dynamic Coherent Risk Measures," Stochastic Processes and Their Applications, 112(2), 185-200. 
Sarin, R., ANd P. WAKker (1998): "Dynamic Choice and Nonexpected Utility," Journal of Risk and Uncertainty, 17(2), 87-119.

SinisCalchi, M. (2001): "Bayesian Updating for General Maxmin Expected Utility Preferences," mimeo.

(2011): "Dynamic choice under ambiguity," Theoretical Economics, 6(3), 379-421.

Strotz, R. (1955): "Myopia and inconsistency in dynamic utility maximization," Review of Economic Studies, 23, 165-180. 